\title{
Standardisation and Product Development in the National Key Economic Area (NKEA) Herbal Sector
}

\author{
Zhari Ismail
}

School of Pharmaceutical Sciences, Universiti Sains Malaysia, 11800 Penang, Malaysia; E-mail: zhari@usm.my

Among the five clusters established to implement the NKEA Herbal Sector, the Standardisation and Product Development Cluster forms an important link to the other clusters namely Agronomy, Discovery, Processing, Preclinical and Clinical Clusters. The aim of this cluster is to ascertain the authenticity, quality, safety and efficacy of the short listed herbs to be developed. The fact that established medicinal plants needs to be propagated, farmed, harvested and further processed into viable healthcare products, special attention is required to the reproducibility of the raw and processed materials in formulating the final product. To ensure that this is implemented, a check list of "must do" items is to be fulfilled in a project proposal under this cluster. Upon fulfilling the items, a prototype product is expected to be achieved in a two year collaborative research and development for the industry partner to take up further for preclinical and clinical studies or develop into products not requiring clinical claims. Currently a number of projects are ongoing and new projects are being solicited in an effort to fulfill the aspirations under the high value herbal sector which highlights the new collaborative approaches involving the researchers and the herbal industry. It is expected with this strategy, a win-win formula among the interested parties will have much to gain in terms of academic applied research and development and the economic gains for the industry.

Keywords: Herbal products, Standardisation, check lists, high value herbs. 